\title{
Generating controllable velocity fluctuations using twin oscillating hydrofoils: experimental validation
}

\author{
S. F. HARDING $\dagger$, G. PAYNE and I. G. BR Y DEN \\ Institute for Energy Systems, University of Edinburgh, \\ Mayfield Road, Edinburgh EH9 3JL, UK
}

(Received ?; revised ?; accepted ?. - To be entered by editorial office)

A method for generating controllable two-dimensional velocity fluctuations using two pitching foils was derived theoretically in a previous companion paper. The present work describes the experimental implementation of the method. The experiments are carried out in a re-circulating water channel optimised to provide low turbulence intensity in the incoming flow. Velocities are measured using an acoustic Doppler velocimeter. The pitching motions of the foils are position-controlled using a closed-loop control system. Two velocity fluctuation patterns are investigated. They consist of a combination of sinusoidal components. Theoretical predictions and experimental measurements are compared in the time and frequency domain. Although some discrepancies are observed, the agreement is generally good and therefore validates the theoretical method for the conditions investigated.

\section{Introduction}

Controlled generation of unsteady water flows in laboratory flumes is a key tool for the experimental investigation of dynamic fluid loads on structures. The approach explored herein is based on flow perturbations generated by two horizontal foils pitching in a uniform velocity stream. The pitching axes of the foils are in the same vertical plane which is perpendicular to the mean flow velocity direction. The two foils are some distance apart and the useful flow perturbations are generated downstream of the foils in the horizontal plane of symmetry of the foil set-up. This approach was pioneered by Ham et al. (1974) and has later also been used by Jancauskas \& Melbourne (1980), Tang et al. (1996), Passmore et al. (2001), Delpero (1992) and Horwich (1993). In these pieces of work, only either longitudinal or vertical perturbations were generated. The present paper deals with the generation of complex combined vertical and longitudinal perturbations.

The theoretical derivation of the relationship between foils motions and generated perturbations is based on a discretized vortex model. In this method, the time series of the foil pitching angles can be used as the input to calculate the time series of the velocity perturbations. This approach is based on the finite summation of the circulation in the wake of the foils. It relies on the assumptions that the foils are assimilated to flat plates pitching at small angles, that the wake is planar, that the bound circulation has negligible effect at the point where velocity perturbations are predicted and finally that the Kutta-Joukowski condition applies at all times. The details of the method are reported in a companion paper (Harding \& Bryden 2012) which also includes preliminary experimental validation against measurements found in the literature (Stapountzis 1982). This preliminary validation only dealt with the case where the foils are pitching in phase

$\dagger$ Email address for correspondence: s.harding@ed-alumni.net 


$\begin{array}{lccc}\text { Screen Type } & \begin{array}{c}\text { Screen Porosity } \\ \left.\text { (Apertures per } \mathrm{cm}^{2}\right)\end{array} & \begin{array}{c}\text { Wire Diameter } \\ (\mathrm{mm})\end{array} & \begin{array}{c}\text { Distance from Inlet } \\ (\mathrm{mm})\end{array} \\ \text { Honeycomb } & 3 & - & 200 \\ \text { Screen 1 } & 16 & 0.56 & 400 \\ \text { Screen 2 } & 30 & 0.25 & 600 \\ \text { Screen 3 } & 40 & 0.35 & 800 \\ \text { Screen 4 } & 90 & 0.25 & 1000 \\ \text { Screen 5 } & 200 & 0.25 & 1200 \\ & \text { TABLE } & \end{array}$

hence generating only vertical perturbations. The work described herein focuses on the validation for more complex perturbation patterns which were generated experimentally using a bespoke apparatus.

The experimental set-up is first described in detail. Combined longitudinal and vertical flow perturbations are generated and the resulting measured velocities are compared with those predicted by the theory.

\section{Experimental set-up}

There are three main components to the experimental set-up: the flow channel, the pitching foil apparatus and the acoustic Doppler instrument for measuring flow velocity.

\subsection{Flume specifications}

The experimental facility used was the recirculating water flume of the University of Edinburgh. This facility incorporates flow conditioning elements which consist of turning vanes and of conditioning screens at the flow inlet. These screens, fabricated from stainless steel mesh, were laid in a cascade of reducing aperture size to gradually decrease the energy of large scale flow disturbances generated at the inlet. The specifications of the screens used are summarised in table 1.

In order to dissipate any wave motion generated during start-up and operation of the flume, a porous beach was installed downstream of the flume outlet.

As a result of this flow conditioning, the turbulence intensity (as defined by Thomson et al. (2010)) was kept under 3\% in the longitudinal direction (Harding 2013).

In order to allow comparison with the theory derived in Harding \& Bryden (2012), it was required that the flow within the test section was two-dimensional. As such, the effect of the transverse boundary layer profile across the flume must be minimised. The boundary layer was modelled according to Prandtl's one-seventh-power law for the development of turbulent boundary layers. The development of the boundary layer thickness, $\delta$, can be expressed as a function of the distance from the inlet, $x$, by Equation 2.1 (Prandtl 1952).

$$
\delta=\frac{0.16 x}{\operatorname{Re}_{x}^{1 / 7}}
$$

The experimental test section was designed to be installed at a location $2.0 \mathrm{~m}$ from the inlet to the flume, where a boundary layer thickness of $\delta=46 \mathrm{~mm}$ is calculated at the flow speed investigated $(U=0.4 \mathrm{~m} / \mathrm{s})$. As such, the test section must be inset from the flume walls by at least this distance for the flow to be two-dimensional. To do this, $6 \mathrm{~mm}$ thick polycarbonate sheets were used to create the test section away from the flume walls. 


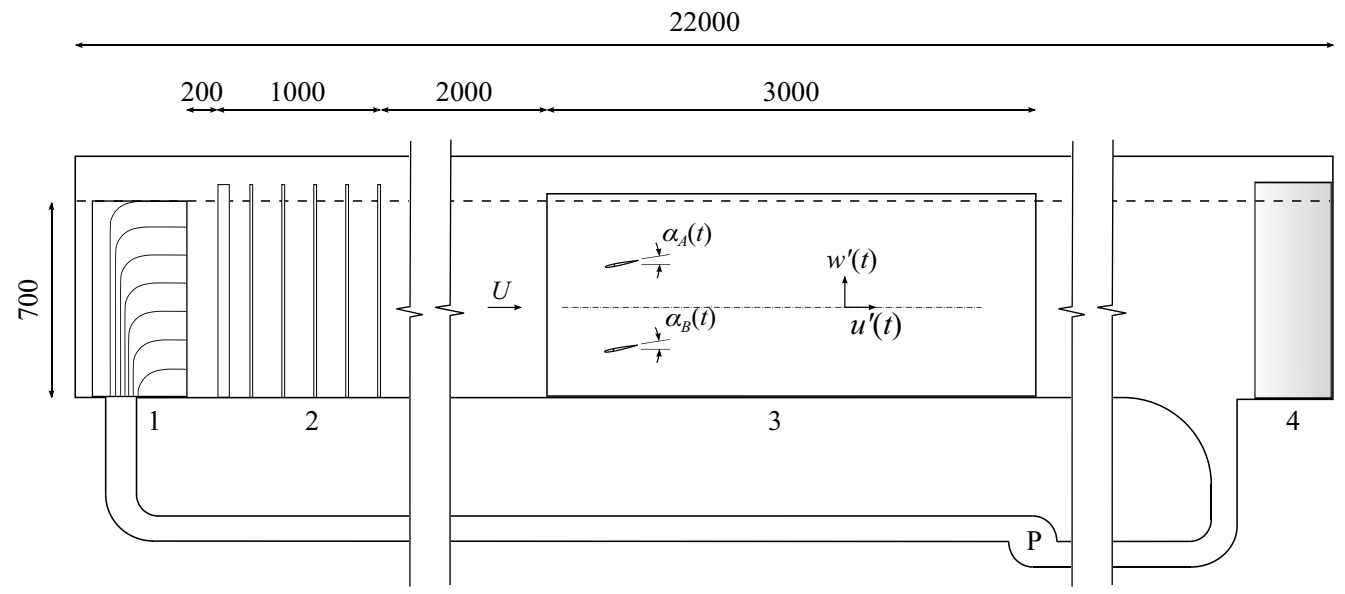

FIGURE 1. Schematic of the flume showing the turning vanes (1), the flow conditioning screens (2), the inset test section (3) and the porous beach (4). All the distances are given in mm.

A new boundary layer will of course develop within the test section. However, with the oscillating foils located $300 \mathrm{~mm}$ from the leading edge of the test section walls, the reset boundary layer is calculated to be less than $\delta=10 \mathrm{~mm}$ by this longitudinal location.

A schematic of the facility is shown in figure 1, indicating the flow conditioning system. The position of the test section, including the pitching hydrofoils, is also shown. A picture of the test section is also shown in figure 2. More details on the flume set-up is described by Harding (2013).

\subsection{Installation of pitching hydrofoils}

The hydrofoils used in the experiment have a NACA-0012 profile, with a chord length of $150 \mathrm{~mm}$ and a span of $250 \mathrm{~mm}$. These were manufactured by rapid-prototyping methods. Each wing rotated about an $8 \mathrm{~mm}$ diameter stainless steel shaft using two push fit polycarbonate bushes.

Each hydrofoil was connected to an independent driving motor by a polyurethane timing belt reinforced with two $0.8 \mathrm{~mm}$ diameter stainless steel cables as shown in figure 2 . The use of two independent driving motors for the two foils allows the phase difference between the foils to be defined by the user. The system was designed so that the belts were located outside of the test section to avoid disturbing the flow conditions, and so ran vertically down the outside of the polycarbonate walls.

Each DC brushless motor is fitted with a magnetic angular position sensor which allows the angle of attack of each foil to be controlled dynamically with a Proportional-IntegralDerivative (PID) feedback loop. This feedback loop was programmed using the FieldProgrammable Gate Array (FPGA) module of a National Instruments CompactRIO. The feedback control was executed using the inbuilt $1 \mathrm{kHz}$ clock, and the foil positions were recorded at a frequency of $50 \mathrm{~Hz}$.

\subsection{Velocity measurement}

Flow velocities were measured using an Acoustic Doppler Velocimeter (ADV). This instrument can measure three dimensional flow properties with a high spatial and temporal resolution. In this study a Nortek Vectrino with the 'Plus' firmware and a down-looking 


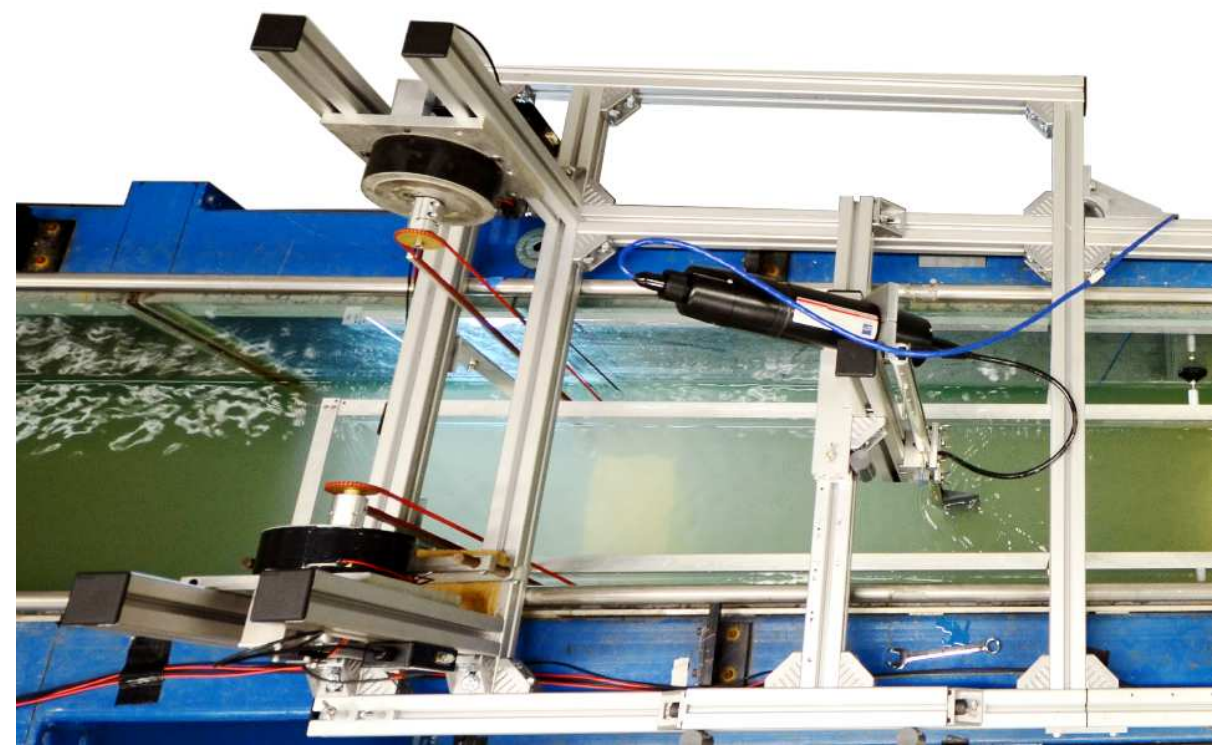

FiguRE 2. Driving motor rig and ADV gantry on top of the recirculating water flume

$\begin{array}{lcc}\text { Setting } & \text { Value } & \text { Unit } \\ \text { Transmit Length } & 1.2 & \mathrm{~mm} \\ \text { Sample Volume } & 4.9 & \mathrm{~mm} \\ \text { Nominal Velocity } & \pm 0.3 & \mathrm{~m} / \mathrm{s} \\ \text { Sampling Frequency } & 200 & \mathrm{~Hz}\end{array}$

TABle 2. Summary of Vectrino settings

cable probe was used. The Nortek Vectrino is capable of measuring the water velocity of a small sample volume in the directions of four converging acoustic beams (Rusello 2009).

In order to accurately and rapidly measure the flow in the flume at a variety of locations, a three degree of freedom gantry system was developed for the flume. This rig used a kinematic positioning system which allows the sample volume to be adjusted in $50 \mathrm{~mm}$ increments in the longitudinal $(x)$, transverse $(y)$ and vertical $(z)$ directions.

The seeding material used in the flume was glass micro-spheres with a mean diameter of $10 \mu \mathrm{m}$. The variance of the density of this seeding material was reduced by allowing $10 \mathrm{~mL}$ of seeding material to settle in a $4 \mathrm{~L}$ vessel of fresh water for $120 \mathrm{~s}$, and siphoning $2 \mathrm{~L}$ of the suspended micro-spheres from a vertical location mid-way up the vessel. This settling time represents the approximate recirculation period of the flume which ensures that vertical drift of the syphoned particles will have a negligible effect on the vertical velocity measured by the ADV. The seeding material was added to the flume at the pump inlet over a period of 120 s for even distribution through the water.

The Vectrino settings used for these experiments are summarised in table 2. They were derived through an extensive sensitivity analysis (Harding 2013) and provide a suitable combination of spatial and temporal resolution and measurement precision for the velocity conditions measured. The sample volume is notionally a circular cylinder with a diameter of $6 \mathrm{~mm}$, and the 'Sample Volume' setting defines the cylinder height. 


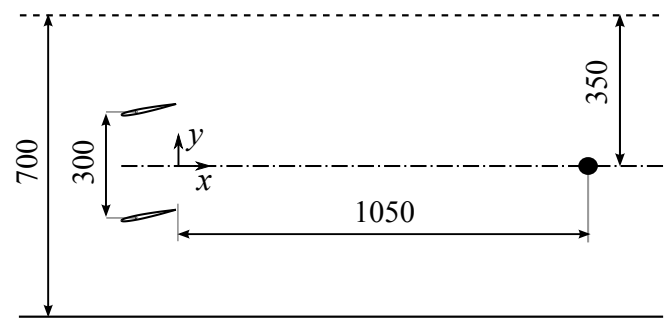

FIGURE 3. Schematic showing the point where velocity is measured (black dot). The bottom of the flume is shown by the solid black line, the water surface by the dashed line and the horizontal plan of symmetry of the foil set-up by the dash-dot line. Dimensions are given in $\mathrm{mm}$.

While the maximum output sampling frequency is $200 \mathrm{~Hz}$, the internal ping rate is significantly greater than this. The setting labelled 'Sampling Frequency' is therefore more accurately described as the output frequency of the ensemble-averaged velocity values. The average number of points per ensemble is equal to $f_{i} / f_{s}$, where $f_{i}$ is the internal sampling frequency or ping rate, and $f_{s}$ is the selected sampling frequency. The internal ping rate for the nominal velocity range of $\pm 0.3 \mathrm{~m} / \mathrm{s}$ selected is $1124 \mathrm{~Hz}$ (Lohrmann 2006).

Under the flow conditions associated with a mean flow velocity of $U=0.4 \mathrm{~m} / \mathrm{s}$, the performance achieved by these settings are a mean correlation of $95 \%$ and a mean signalto-noise ratio of $20 \mathrm{~dB}$.

\section{Experiments}

\subsection{Location of velocity measurements}

In the theoretical solution described in Harding \& Bryden (2012), the spatial range L of the circulation summation was investigated up to the value $L=30 c$, where $c$ is the foil chord length. It was found that using a reduced range of $L=14 c$ yielded velocity perturbation values which were within only $2 \%$ of the magnitude of those obtained with $L=30 c$. This reduced range offers the practical advantage of a shorter test section at the expense of only a small loss in precision. The length of the test section was therefore set to safely accommodate a summation range of $L=14 \mathrm{c}$. The longitudinal location for velocity measurements was set to $x=7 c$; at the midpoint of the summation range. This corresponds to a horizontal distance of $1050 \mathrm{~mm}$ downstream of the tail edge of the pitching foils. Velocities were measured in the horizontal plane of symmetry of the foil set-up which corresponds to a water depth of $350 \mathrm{~mm}$. The ADV was positioned halfway through the width of the water channel. The location of the measurement point is shown on figure 3 .

\subsection{Multiple frequency two-dimensional velocity fluctuations}

In order to demonstrate the flexibility of the configuration to generate user-defined velocity perturbations independently in both directions, two arbitrary foil motions were defined using a number frequency components. These are referred to as Waveform 1 and Waveform 2. The frequencies of all the harmonics used to specify Waveform 2 are half these of Waveform 1. The time series of the foil pitch angles, $\alpha$, input to the vortex model and to the experimental system are shown in figure 5. All the experiments were performed at a flow speed $U=0.4 \mathrm{~m} / \mathrm{s}$, corresponding to $R e=5.8 \times 10^{4}$. 


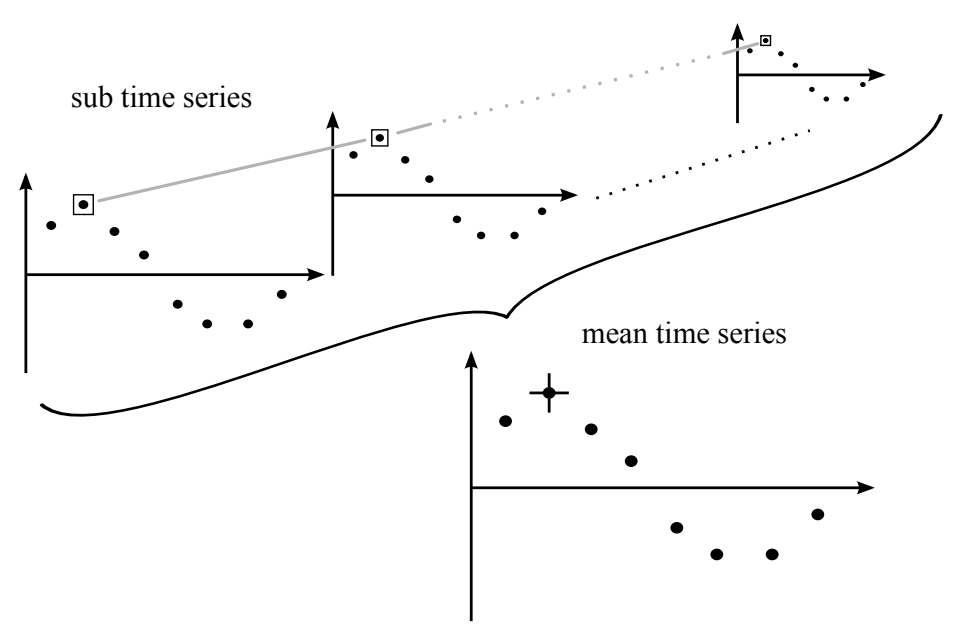

FIGURE 4. Schematic of the data processing used to compute the mean velocity time series. The grey line symbolises the averaging process across the different sub time series for the data point surrounded by a square which results in the data point marked by a cross on the mean time series.

The velocity perturbations were acquired for a period of 300s with the ADV sampling frequency set at its maximum of $200 \mathrm{~Hz}$. In post-processing, the time series were divided into shorter time series whose duration corresponded to the period of the respective waveform (10s for Waveform 1 and 20s for Waveform 2). This led to 30 shorter 'sub time series' for Waveform 1 and 15 for Waveform 2. For each time step of the sub time series, velocity values were averaged across the different sub time series yielding the mean velocity for that time step. This data processing is illustrated by figure 4 . This resulted in a mean velocity time series for each waveform which are plotted on figure 5 where they are compared with the waveforms predicted by the vortex model. The velocity fluctuations in the longitudinal and vertical directions are denoted as u' and w', respectively. Given that the ADV sampling frequency used was the same for both waveforms, the averaging process is carried out over a smaller number of periods for the longer duration waveform

\section{Results analysis}

For both waveforms, it can be seen that the spread of the experimental points is significantly greater in the longitudinal direction than the vertical one. This can be attributed to the geometric configuration of the transducers in the Nortek Vectrino. These are angled at $60^{\circ}$ to the transmitted beam direction. This results in a higher precision for readings in the vertical direction which is more aligned with the receivers than the longitudinal flow direction.

The agreement between experimental measurements and theoretical predictions is generally good, with some discrepancies. These are more noticeable for Waveform 1 in the vertical direction and Waveform 2 in the longitudinal direction. For the latter, the theoretical time series predicts higher values than the measurements at the two main peaks.

In order to investigate further the discrepancies between experimental and theoretical results, a Fourier analysis is carried out. Figure 6 shows comparison for the amplitude of the frequency components. Although the Nyquist frequency is $100 \mathrm{~Hz}$, the frequency 

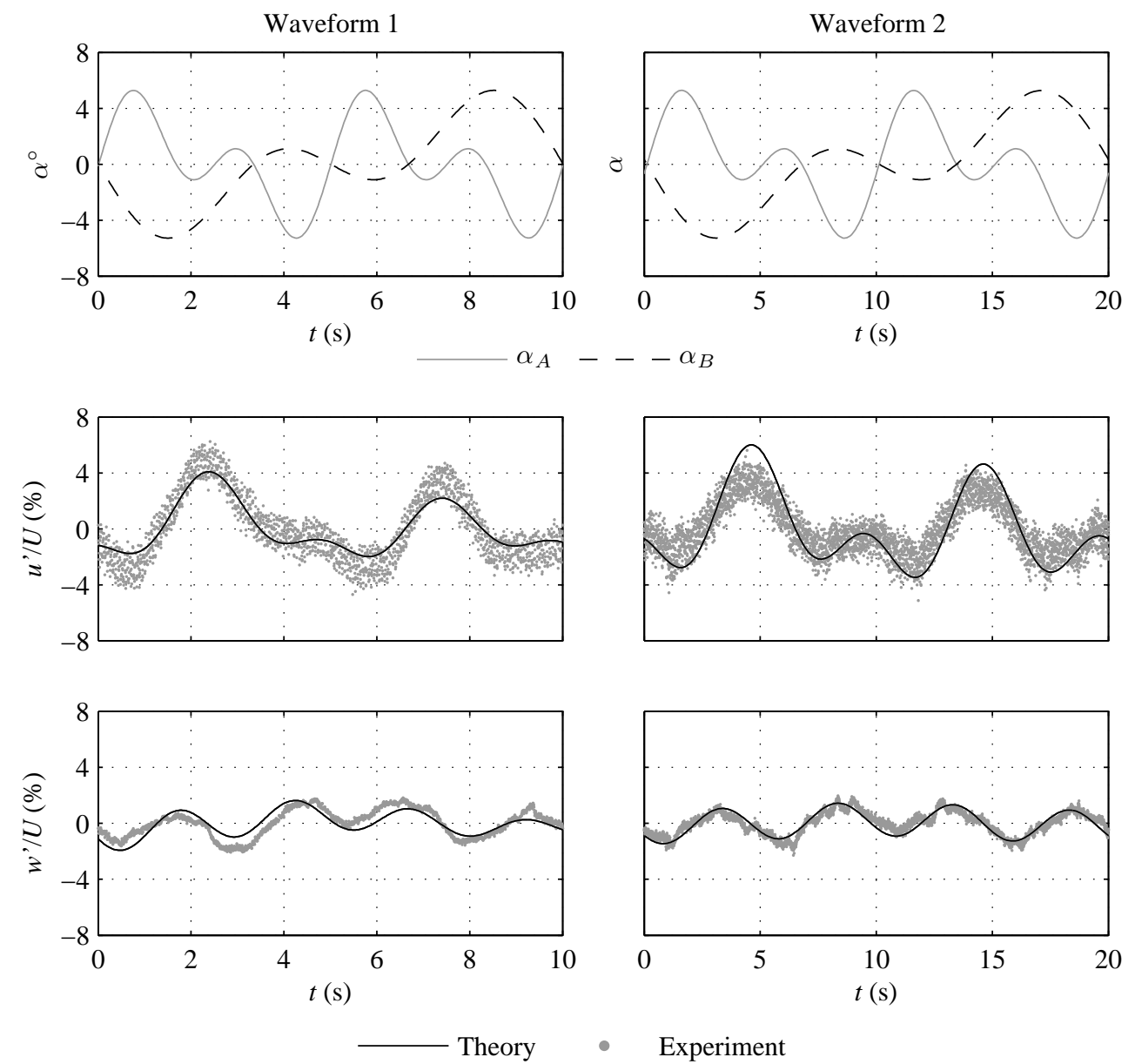

FIGURE 5. Experimental results of two-dimensional flow control using twin pitching foils. The pitch motion of the foils is defined in the top row of plots. The results of Waveform 1 are presented in the left-hand column, and the results of Waveform 2 are presented in the right-hand column. The velocity perturbations are normalised by the mean longitudinal flow velocity, $U$.

range on the figure is adjusted to focus on the main frequency components of the signal. This improves clarity and is thought appropriate as no other frequency peak was recorded beyond what is shown.

For both waveforms, there is no frequency spillage except potentially for the first component of the longitudinal velocity perturbation of Waveform 2, although that could also be an artefact of signal noise. The frequencies of the velocity perturbations generated experimentally therefore correspond very accurately to those predicted by the theory.

In terms of frequency component amplitude, the match between experimental and theoretical is generally good except for the longitudinal velocity perturbations of Waveform 2 where the theory tends to over-predict what is generated experimentally. This is consistent with what can be observed on figure 5 where the theoretical line exceeds the experimental measurements for the two main peaks of the waveform.

One can also notice that for both waveforms, the measured vertical velocity perturbation exhibits a frequency component at the same frequency as that of the second component of the longitudinal velocity perturbation. This is not predicted by the the- 

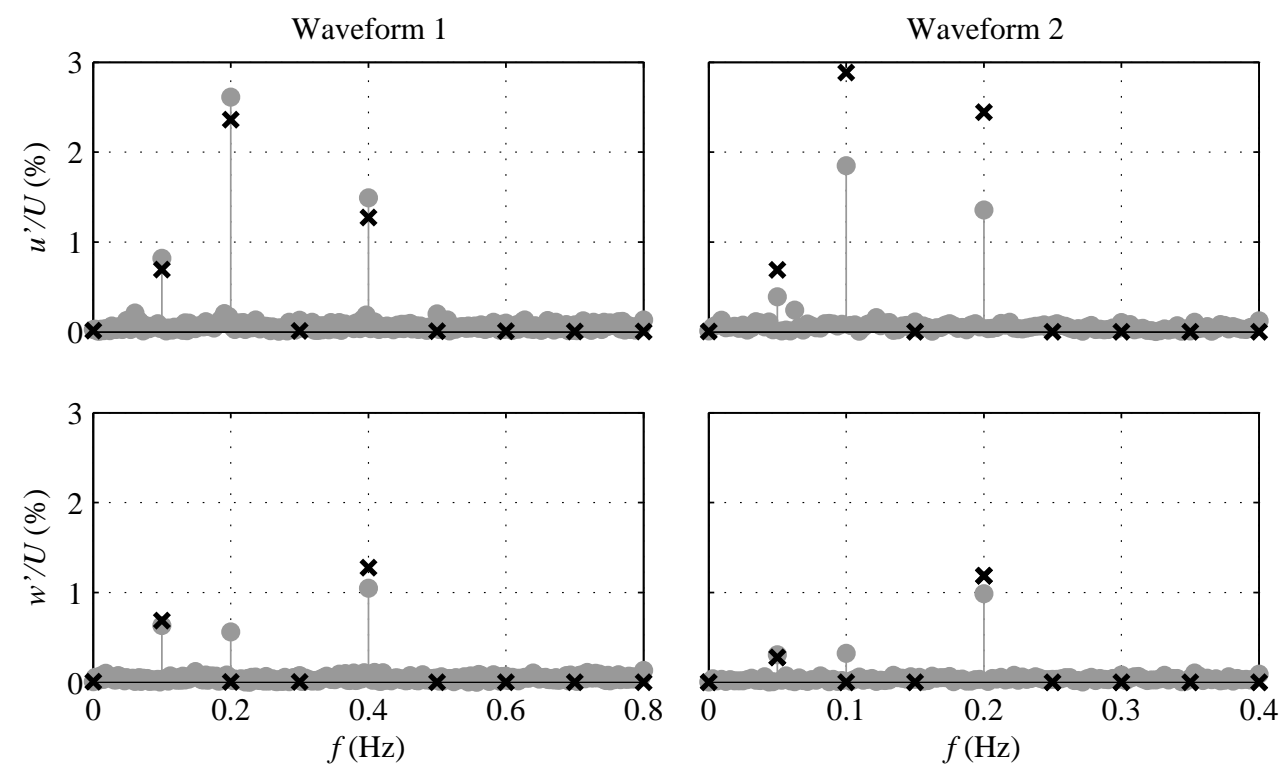

$\mathbf{x}$ Theory $\longrightarrow$ Experiment

FiguRE 6. Results of Fourier analysis of both experimental and theoretical velocities generated by the foil pitch motions defined by Waveform 1 and Waveform 2 in figure 5 .

ory. It is not believed to be a harmonic of the first component of the vertical velocity perturbation for two reasons:

- For both waveforms, the amplitude is similar to that of the first component whereas the amplitude of harmonics is usually significantly lower than that of the fundamental.

- When looking at the third vertical component (at $0.4 \mathrm{~Hz}$ and $0.2 \mathrm{~Hz}$ for Waveforms 1 and 2 respectively), it can be seen that these do not produce any significant harmonics at $0.8 \mathrm{~Hz}$ and $0.4 \mathrm{~Hz}$ (for Waveforms 1 and 2 respectively). It is therefore unlikely that the first vertical components (at $0.1 \mathrm{~Hz}$ and $0.05 \mathrm{~Hz}$ for Waveforms 1 and 2 respectively) would generate harmonics at $0.2 \mathrm{~Hz}$ and $0.1 \mathrm{~Hz}$ (for Waveforms 1 and 2 respectively) whose amplitudes would be as high as those observed.

A possible explanation is that the high amplitude components in one direction affect the components in the perpindicular direction at the same frequency. It can indeed be observed that the second longitudinal components (at $0.2 \mathrm{~Hz}$ and $0.1 \mathrm{~Hz}$ for Waveforms 1 and 2 respectively) have the highest amplitude and it is at these frequencies that the most significant 'cross-talk' between longitudinal and vertical components takes place. A possible physical explanation for this observation is that the theory used to predict the velocity perturbations assumes planar wake (Harding \& Bryden 2012). When the path followed by vortices start to depart from the horizontal planes downstream of the foils, the longitudinal and vertical velocity components they induce is not fully accounted for by the theory. Moreover, the stronger the vortex, the larger the induced velocity; which would make this phenomenon more noticeable for stronger vortices and hence for larger amplitude velocity components. 


\begin{tabular}{c|ccc|ccc} 
& \multicolumn{3}{|c|}{ Waveform 1 } & \multicolumn{3}{c}{ Waveform 2} \\
Frequency $(\mathrm{Hz})$ & 0.1 & 0.2 & 0.4 & 0.05 & 0.1 & 0.2 \\
\hline \multirow{2}{*}{$w$} & - & -0.00944 & 19.0 & - & -0.360 & 0.476 \\
$w$ & - & - & 31.3 & - & - & 8.42 \\
$u-w$ & -27.4 & - & -15.0 & -23.2 & - & -15.2
\end{tabular}

TABLE 3. Phase shift comparison, in degrees, between experimental measurements and theoretical predictions.

Table 3 shows the phase comparison between the measured signal and what is predicted by the theory for both waveforms. These are calculated as follows:

- Phase shift are calculated between the first frequency component and the subsequent ones for both directions (longitudinal and vertical) independently. In other words, for each direction the phase angle of all but the first frequency components are calculated with respect to the first frequency component. This is done both for the experimental and theoretical time series.

- Theoretical phase shifts obtained this way are then subtracted from the corresponding experimental phase shifts to assess the discrepancies between theoretical and experimental results.

- In addition, phase shifts are calculated between longitudinal and vertical velocity perturbations for each frequency components. This is done both for the experimental and theoretical time series. As previously, the theoretical phase shifts are subtracted from the corresponding experimental ones.

Table 3 therefore gives an indication of how well the theoretical method predicts the phase shift between the different frequency components in the same direction and between the same frequency components but across the different directions. It should be noted that there are only two frequency components predicted by the theory for the vertical velocity. Although a third component was measured experimentally, its phase cannot be compared to any theoretical value, hence its absence in the table.

The maximum discrepancy in phase shift between experimental measurements and theoretical predictions is $31.3^{\circ}$. In terms of phase shift between the different frequency components of a same direction, the discrepancies are smaller for Waveform 2 with a very good agreement in the longitudinal direction. For both waveforms, discrepancies are larger in the vertical direction than in the longitudinal one. In terms of phase shift between velocity components of the same frequency but across directions, the discrepancies are very similar for both waveforms. This suggests that the underlying physical phenomenon causing these differences is consistent across both experiments and therefore weakly affected by the frequency of the velocity perturbation components.

\section{Conclusions}

Following the theoretical derivation, in a companion paper (Harding \& Bryden 2012), of a method for generating arbitrary velocity fluctuations with two oscillating hydrofoils, an experimental implementation of the method has been carried out.

Prior to the experiments, the flow facility has been optimised for controlling incoming 
turbulence intensity and boundary layer effects which are not accounted for by the theoretical model. The resulting experimental set-up with its measurement apparatus have been described in details.

Two complex combinations of longitudinal and vertical velocity perturbations have been experimentally generated and measured. These waveforms consist of several sinusoidal components of different frequencies and amplitudes.

Comparison between theoretical predictions and experimental measurements have been carried out in the time and frequency domains. The agreement is generally good. In terms of amplitude, some discrepancies are observed, mainly in the longitudinal direction for Waveform 2. A phenomenon of 'cross-talk' between longitudinal and vertical directions which is not predicted by the theory has also been identified in the experimental measurements for the largest amplitude frequency components.

The phase analysis of the results shows that for phase shifts between the different frequency components along a same direction, the agreement between theory and experiments is better in the longitudinal direction.

Although some discrepancies exist, the overall good agreement between the theoretical predictions and the measurements provide an experimental validation of the method derived by Harding \& Bryden (2012) for the flow conditions investigated. Further experimental investigation will be required to explore the limits of applicability of the method in terms of flow velocity and pitching angles and frequencies. However, the validation described herein already opens up a large range of possibilities for the experimental investigation of complex two-dimensional velocity fluctuation patterns and their dynamic effects on structures.

\section{REFERENCES}

Delpero, P. M. 1992 Investigation of flows around a two dimensional hydrofoil subject to a high reduced frequency gust loading. Master's thesis, Massachusetts Institute of Technology.

Ham, N. D., Bauer, P. H. \& Lawrence, T. L. 1974 Wind tunnel generation of sinusoidal lateral and longitudinal gusts by circulation control of twin parallel airfoils. Tech. Rep. 137547. NASA Contractor Report.

HARDing, S.F. 2013 Unsteady velocities of energetic tidal currents: an investigation into dynamic flow effects on lifting surfaces at field and experimental scale. PhD thesis, University of Edinburgh.

Harding, S.F. \& Bryden, I. 2012 Generating controllable velocity fluctuations using twin oscillating hydrofoils. Journal of Fluid Mechanics 713, 150-158.

Horwich, E. A. 1993 Unsteady response of a two-dimensional hydrofoil subject to high reduced frequency gust loading. Master's thesis, Massachusetts Institute of Technology.

Jancauskas, E.D. \& Melbourne, W.H. 1980 The measurement of aerodynamic admittance using discrete frequency gust generation. In 7th Australasian Hydraulics and Fluid Mechanics Conference. Brisbane.

Lohrmann, A. 2006 Vector ping rate. Nortek AS Knowledge Center, Accessed 23rd Nov, 2012.

Passmore, M.A., Richardson, S. \& Imam, A. 2001 An experimental study of unsteady vehicle aerodynamics. Proceedings of the Institution of Mechanical Engineers, Part D: Journal of Automobile Engineering 215, 779-788.

Prandtl, L. 1952 Essentials of Fluid Mechanics. Blackie and Son.

Rusello, P. 2009 A practical primer for pulse coherent instruments. Tech. Rep. 027. Nortek.

StAPOUNTZIS, H. 1982 An oscillating rig for the generation of sinusoidal flows. Journal of Physics E: Scientific Instruments 15, 1173-1176.

Tang, D.M., Cizmas, P.G.A. \& Dowell, E.H. 1996 Experiments and analysis for a gust generator in a wind tunnel. Journal of Aircraft 33 (1), 139-148.

Thomson, J., Polagye, B., Richmond, M. \& Durgesh, V. 2010 Quantifying turbulence for tidal power applications. In OCEANS 2010. Seattle, Washington. 